\title{
Fogitare
}

\section{INCIDENTES EM SESSÕES DE HEMODIÁLISE À BEIRA LEITO EM UNIDADES DE TERAPIA INTENSIVA}

\section{INCIDENTS IN BEDSIDE HEMODIALYSIS SESSIONS IN INTENSIVE CARE UNITS}

\author{
Natália Nunes Costa ${ }^{1}$ \\ Regiane dos Santos Soares Barreto ${ }^{1}$ (i) \\ Matheus Martins da Costa ${ }^{1}$ (1) \\ Raquel Machado Schincaglia ${ }^{1}$ (i) \\ Nara Rúbia de Freitas ${ }^{1}$ (i) \\ Cristiana da Costa Luciano ${ }^{1}$ (1) \\ Karina Suzuki ${ }^{1}$ (i)
}

\begin{abstract}
Objective: to analyze incidents during bedside hemodialysis sessions held in Intensive Care Units. Method: cross-sectional, retrospective study, developed in Intensive Care Units of Goiânia, Brazil. Analysis of incidents identified in bedside hemodialysis sessions was performed between March and April 2018. Descriptive analysis was performed for categorical data and for continuous data, mean and standard deviation of the mean. Results: 873 sessions were analyzed, and 563 incidents were recorded. Among those reported, 259 (46\%) were inherent to the treatment. Near miss was the most frequent type of incident, generating delay in the beginning of the session, and no support from the multi-professional team (p<0.05). In $132(56.12 \%)$ of the notifiable circumstances, the professional worked for more than 12 uninterrupted hours $(p<0.05)$. Conclusion: the analysis of the results allows students and experts in the area to subsidize improvement strategies for the service.
\end{abstract}

DESCRIPTORS: Quality of Health Care; Sentinel Surveillance; Patient Safety; Patient Harm; Nephrology Nursing. 
Os incidentes em saúde são eventos ou circunstâncias que podem resultar em dano ao paciente. Ocorrem todos os dias nos diversos cenários e estabelecimentos de saúde, e podem gerar lesão, sofrimento, incapacidade e óbito ${ }^{(1-2)}$.

A Organização Mundial de Saúde (OMS) classifica os incidentes em circunstâncias notificáveis, com potencial significativo para dano: near miss ou quase erro, quando não atingiu o paciente; incidente sem danos, o qual atingiu o paciente, todavia não gerou dano; e incidente com dano ao paciente, conhecido como Evento Adverso $(E A)^{(1)}$.

Os níveis assistenciais de maior complexidade, tais como a Unidade de Terapia Intensiva (UTI), são propícios à ocorrência de incidentes. A criticidade exige o uso de tecnologias de saúde complexas, e a urgência na tomada de decisões de risco, muitas vezes associada ao déficit de informações sobre o histórico do paciente, torna-o vulnerável a danos ${ }^{(3)}$.

Dentre os tratamentos invasivos comumente realizados em pacientes em UTI, a hemodiálise (HD) à beira leito é bastante comum ${ }^{(4)}$. O número absoluto, taxas de incidência e prevalência em tratamento hemodialítico crescem constantemente ${ }^{(5)}$. Portanto, o serviço de HD necessita de vias de redução de incidentes e mitigação de danos.

Estudo escocês, em pacientes em HD em UTI, identificou 9,6\% de óbitos causados por infecções e 3,5\% relacionados a erros organizacionais e humanos, como decisões médicas inadequadas, desconhecimento no manejo da hipercalemia e das prescrições, e insuficiência tecnológica para intervenção nos acessos vasculares ${ }^{(6)}$.

No Brasil, estudo realizado com portadores de Doença Renal Crônica (DRC) identificou $1.272 \mathrm{EA}$. Os mais prevalentes foram: fluxo sanguíneo inadequado, sangramento pelo acesso venoso e coagulação do Sistema Extracorpóreo (SE). Desses EA, 0,9\% foram graves e $0,8 \%$ geraram óbito ${ }^{(7)}$.

Para a redução dos EA, é necessário entender quais sãos as causas e fatores contribuintes $^{(8)}$. Contudo, a subnotificação é uma realidade e um problema de nível local e nacional(9).

A equipe de enfermagem possui papel chave na segurança do paciente, pois atua na identificação, gerenciamento e proposição de planos capazes de reduzir significantemente incidentes ${ }^{(10)}$.

Nessa perspectiva, este estudo, pioneiro no Brasil, objetivou analisar incidentes ocorridos durante sessões de HD à beira leito na UTI.

\section{MÉTODO}

Estudo de caráter transversal, realizado em UTI na cidade de Goiânia-GO, Brasil, no ano de 2018. Nessa cidade existem doze clínicas que realizam serviço terceirizado de HD com atendimento em UTI. Dentre essas, foi escolhida, por conveniência, uma clínica com atendimento em dez UTI de instituições privadas destinadas ao atendimento de pacientes adultos para compor a amostra deste estudo.

A clínica em questão oferece serviço por meio de uma equipe de cinco médicos nefrologistas, um enfermeiro nefrologista supervisor e dez técnicos em enfermagem. Os 
técnicos realizam e acompanham todas as sessões de HD à beira leito, exercendo atividades em escala de doze horas de trabalho para 36 horas de descanso.

Para cada sessão de HD, eram encaminhados insumos ao local: uma máquina de HD e uma de tratamento de água, conhecida como osmose reversa portátil, além de um técnico em enfermagem. O enfermeiro da clínica tem por função supervisionar a distância, por meio de telefone, além de realizar supervisão presencial com visitas diárias. O enfermeiro da UTI contribui para a supervisão dentro da unidade onde são realizadas as sessões de HD.

$\mathrm{Na}$ UTI, são realizados diferentes tipos de sessões de HD: a convencional, a ultra filtração isolada, a HD contínua e a sustained low efficiency dialysis (SLED), conforme a prescrição da equipe médica de nefrologistas.

A fonte dos dados para esse estudo foram as notificações realizadas nos meses de março e abril do ano de 2018, pela equipe de enfermagem na ficha de prescrição de HD à beira leito em UTI preenchidas ao final de cada sessão, independentemente de ter havido incidente. Foi realizada a leitura de todas as notificações realizadas no período do estudo, seguida da transcrição dessas notificações para instrumento semiestruturado, elaborado pela pesquisadora para a coleta dos dados notificados.

O instrumento elaborado para a transcrição dos dados foi construído com base no Minimal Information Model for Pacienty Safety Incident Reporting and Learning Systems ${ }^{(8)}$. Nesse instrumento, anotou-se informações sobre a sessão de HD (motivo de HD, fonte pagadora, modalidade de HD, uso de heparina, tempo prescrito e tempo decorrido de $\mathrm{HD}$, atraso no início/término, interrupção da sessão); sobre os incidentes (tipos e motivos); sobre o profissional que realizava a sessão (horas de trabalho, número de sessões realizadas no dia, apoio da equipe nas sessões); e também informações sobre o indivíduo que sofreu o incidente (sexo, idade, comorbidades, acesso para HD).

Os dados coletados foram digitados no programa Microsoft Excel $\bigotimes^{\circledR}$. A primeira análise foi a leitura das notificações para classificá-las, e a digitação foi feita de modo que cada incidente fosse caracterizado como uma observação. Em seguida, as notificações foram agrupadas segundo a classificação internacional de segurança do paciente da OMS ${ }^{(1)}$, em circunstâncias notificáveis, near miss, incidente sem danos e EA.

Os eventos adversos foram divididos em eventos adversos inerentes ao tratamento, quando não era evidente a falha nos processos de assistência e de tecnologias de saúde, e eventos adversos evitáveis, quando o dano ocorreu por falhas evitáveis durante o processo. Posteriormente foi realizada análise descritiva para os dados categóricos, apresentados em frequências absolutas ( $n$ ) e relativas (\%), e para os dados contínuos em média e desviopadrão. Para testar a homogeneidade dos grupos em relação às proporções, foi utilizado o Teste Exato de Fisher Bicaudal.

Foi realizado o teste de Shapiro-Wilk para testar a normalidade dos dados contínuos e, em seguida, procedeu-se com o teste $U$ de Mann-Whitney para comparar os dados contínuos na ausência de normalidade. O nível de significância utilizado para todos os testes foi de $5 \%$. Foi utilizado o software STATA $®$ versão 14.0 nesta análise.

Essa pesquisa foi aprovada em Comitê de Ética em Seres Humanos e possui Certificado de Apresentação para Apreciação Ética, sob parecer n. 1.922.585. 
Dessas, 563 (89,79\%) apresentavam registro de incidentes.

total de incidentes notificados foi de 563, sendo a maioria em pacientes do sexo masculino 305 (54,17\%), em sessões de HD sem motivo descrito 289 (54,22\%), portadores de diabetes 115 (45,63\%), em pacientes com convênio não-SUS 532 (94,49\%), que realizavam HD por meio de cateter 500 (88,81\%), modalidade SLED 384 (68,33\%), sem heparina 497 $(88,28 \%)$ e em terapias com tempo prescrito igual ao realizado $451(80,11 \%)$.

Dentre o total de incidentes notificados, a categoria de maior frequência foi incidente inerente ao tratamento, com 259 (46,00\%) relatados. Especificamente para esse tipo de incidente, há destaque para alterações clínicas durante as sessões em 181 (32,14\%) das ocorrências, sendo a mais frequente delas a hipotensão (63,09\%). A categoria outros sinais e sintomas foi composta por hiperglicemia $(0,69 \%)$, cianose $(0,69 \%)$ e rebaixamento do nível de consciência (1,37\%) (Figura 1).

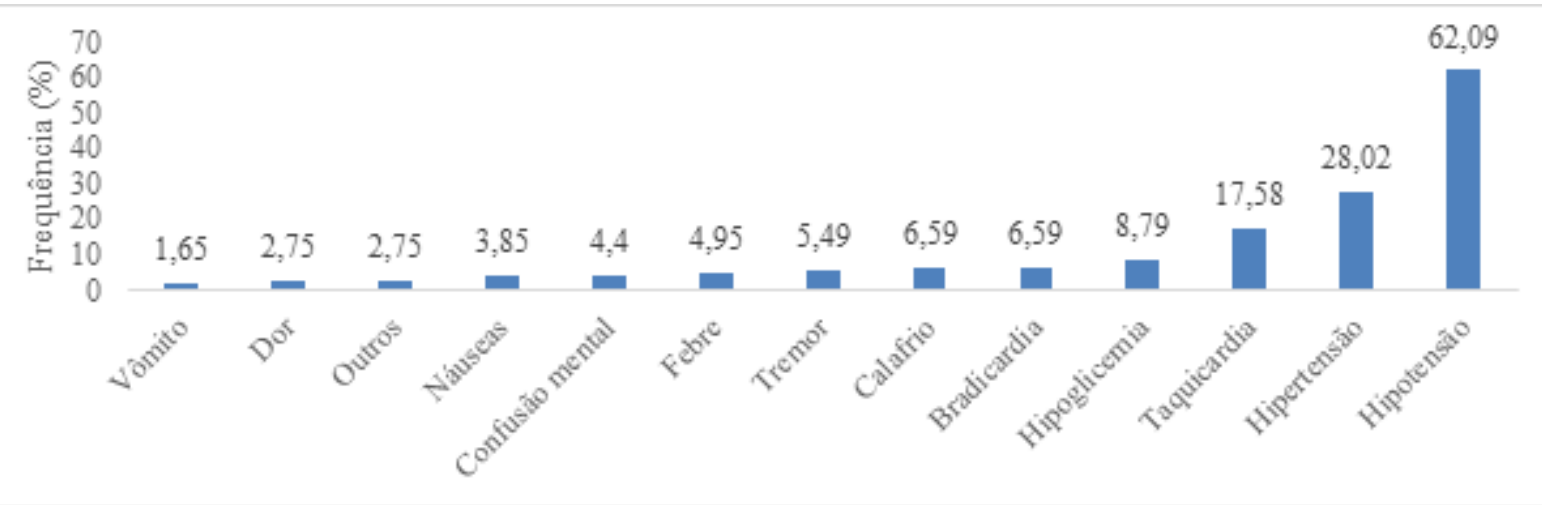

Figura 1 - Frequências dos 181 Incidentes relacionados aos sinais e sintomas dos pacientes em hemodiálise à beira leito em UTI. Goiânia, GO, Brasil, 2018. Fonte: Autores (2018)

A segunda categoria de incidentes com mais notificações foi relacionada às tecnologias de saúde, 158 (28,06\%), com destaque para falhas de tecnologias de saúde da clínica 105 $(66,46 \%)$, das quais $41(39,05 \%)$ foram relacionadas ao vazamento de água na osmose reversa. Foi relevante também a frequência de notificações de incidentes relacionados à prestação do cuidado $115(20,43 \%)$, sendo mais frequentes os incidentes relacionados ao uso do cateter para HD, 51 (44,35\%). A maior frequência foi de incompatibilidade do acesso com o fluxo prescrito: $44(86,28 \%)$ dos incidentes notificados nessa categoria.

Houve 29 (5,15\%) notificações de incidentes em que não havia relatos dos motivos, não sendo possível realizar sua classificação quanto ao tipo. Todos os incidentes identificados foram classificados segundo a nomenclatura da $\mathrm{OMS}^{(1)}$ e apresentados na Tabela 1.

Tabela 1 - Classificação dos incidentes notificados em sessões de hemodiálise à beira leito em Unidades de Terapia Intensiva. Goiânia, GO, Brasil, 2018

\begin{tabular}{lcc} 
Classificação do incidente & $\mathbf{n}$ & $\%$ \\
\hline Circunstância notificável & 235 & 41,74 \\
\hline
\end{tabular}




\begin{tabular}{lcc}
\hline Eventos adversos & 312 & 55,41 \\
\hline Evento adverso inerente ao tratamento & 223 & 39,61 \\
\hline Evento adverso evitável & 89 & 15,81 \\
\hline Near miss ou quase erros & 10 & 1,78 \\
\hline Incidente não descrito & 6 & 1,06
\end{tabular}

Fonte: Autores (2018)

Os EA totalizaram $312(55,41 \%)$ dos incidentes notificados, e foram divididos em EA inerentes ao tratamento e EA evitáveis. Os EA inerentes ao tratamento se destacaram, 223 $(39,61 \%)$, devido principalmente a sinais e sintomas apresentados pelo paciente, como hipotensão, hipertensão e taquicardia.

Já os EA evitáveis, representando 89 (15,81\%) dos incidentes relatados, foram devido, principalmente, às falhas em tecnologia em saúde. E os near miss, ou quase erros, de maior ocorrência foram programação da máquina de HD equivocada, principalmente com relação ao fluxo do dialisato. Em seis $(1,06 \%)$ incidentes, não havia informações suficientes para classificar o incidente conforme a nomenclatura da OMS ${ }^{(1)}$.

Em 335 (53,43\%) das 627 notificações avaliadas, os técnicos estavam trabalhando há mais de 12 horas contínuas e em 528 (84,21\%) já haviam realizado pelo menos três sessões de HD naquele dia. Foi observada associação significativa entre o tempo maior que 12 horas de trabalho pelo técnico responsável pela sessão, e circunstância notificável $(p<0,001)$. Já EA evitável e EA inerente ao tratamento foram estatisticamente associados com tempo de trabalho menor que 12 horas, com $p=0,005$ e $p<0,0001$, respectivamente (Figura 2).

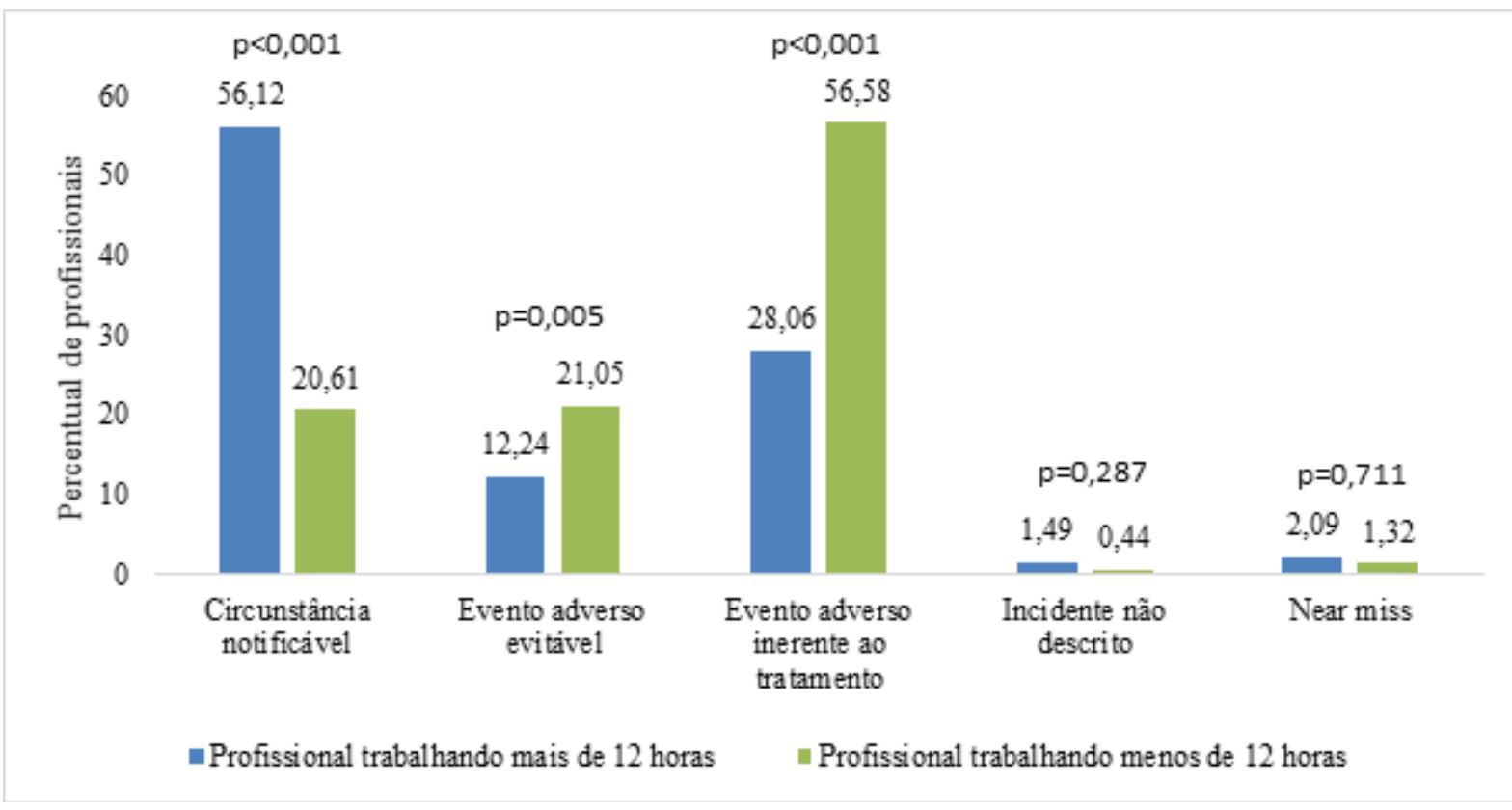

Figura 2 - Relação entre tempo de trabalho ininterrupto e classificação do incidente. Goiânia, GO, Brasil, 2018. Fonte: Autores (2018) 
Dentre as circunstâncias notificáveis, foi observado nas 627 notificações que houve maior frequência de atraso na sessão $(16,42$ versus $4,79 \%, p<0,001)$; maior frequência de interrupção momentânea da sessão $(12,24 \%$ versus $6,16 \%, p=0,009)$, maior frequência de atraso no término da sessão $(3,88 \%$ versus $1,03 \%, p=0,039)$ e, por fim, maior frequência de apoio por parte da equipe $(25,08 \%$ versus $17,20 \%, p=0,001)$ nas sessões conduzidas por técnicos que estavam trabalhando há mais de 12 horas, em comparação com aqueles que não estavam trabalhando por mais de 12 horas.

Considerando a ocorrência de notificação como variável de desfecho, foram estatisticamente significantes os incidentes notificados em sessões ainda sem indicação do motivo ( $p=0,015)$, em sessões por convênio não-SUS $(p=0,049)$, na HD convencional $(p<0,001)$ e em sessões em que a relação entre tempo de sessão prescrito e realizado foi o mesmo $(p=0,020)$. Por outro lado, houve menor frequência de incidentes quando a HD foi realizada na modalidade SLED $(p=0,009)$ e contínua $(p=0,013)$.

\section{DISCUSSÃO}

Os dados de análises de ocorrências de incidentes em pacientes graves e com maior invasão terapêutica evidenciam um cenário alarmante para a segurança do paciente ${ }^{(2)}$. Os resultados são, portanto, indicadores que direcionam os olhares da gestão de saúde para ações de qualidade e segurança no serviço. Isso faz-se necessário pois, segundo Relatório Bienal da International Society of Nephrology (ISN) ${ }^{(11)}$, entre 8 e $10 \%$ da população adulta do mundo apresenta algum tipo de lesão renal, podendo vir a necessitar de atendimentos de alta complexidade.

Estudo realizado no Brasil sugere que o sexo do paciente pode refletir subjetivamente na carga de trabalho da equipe de enfermagem, como por exemplo o peso dos pacientes homens, comumente superior ao das mulheres, mesmo que isso não seja uma regra ${ }^{(12)}$.

Diante desse perfil, para a segurança do paciente, é necessário promover a constante capacitação dos profissionais, principalmente no manejo da doença renal aguda (DRA) em pacientes acima de 60 anos $^{(11)}$. $O$ conhecimento da idade do paciente permite a gestão dos recursos humanos, uma vez que pacientes acima de 60 anos demandam cuidados diferenciados e são suscetíveis a incidentes ${ }^{(12)}$.

A não descrição do diagnóstico na prescrição de HD foi justificada pela complexidade do paciente admitido em UTI com emergência para o tratamento de HD à beira leito, sendo esta prescrita com base em sinais e sintomas clínicos, antes da conclusão do diagnóstico.

A maioria das sessões de HD à beira leito foram realizadas por meio de convênio de saúde não-SUS. Isso ocorre em virtude das UTI onde a pesquisa foi realizada não serem de serviços de saúde públicos, e sim de instituições particulares que possuem convênio com o SUS.

Países em desenvolvimento devem investir na gestão da qualidade e segurança da assistência ao paciente, pois os dados acima descritos constatam as lacunas dos processos de saúde em serviços de HD, que associados ao perfil dos pacientes evidenciam o risco do paciente que consegue acesso ao tratamento de HD vir a óbito, vítima de um incidente.

Os incidentes ocorreram em maior frequência na realização da modalidade de terapia HD SLED. Estudo realizado no Brasil, em UTI, aponta a HD convencional como modalidade de HD mais prescrita e, portanto, com maiores chances de incidentes ${ }^{(5)}$.

O presente estudo identificou com maior frequência a hipotensão, classificada nessa pesquisa como incidente inerente ao tratamento. Pesquisa realizada em unidade 
de referência em HD no Brasil apontou que pacientes internados que realizavam HD apresentavam instabilidade hemodinâmica devido ao uso de drogas vasoativas. Identificou, ainda, que pacientes apresentaram hipotensão, hipertensão e hipoglicemia, evoluindo parada cardiorrespiratória ${ }^{(13)}$.

Estudo realizado no Brasil refere a hipotensão como principal complicação da HD, ocorrendo pico de pressão arterial em $25,5 \%$ das complicações identificadas ${ }^{(14)}$. Atualmente, os tratamentos de HD contam com aparelhos de diálise que auxiliam na segurança do paciente, com tecnologia avançada que ajuda na prevenção da hipotensão, com mecanismos de controle da UF, permitindo variar a concentração de sódio do dialisato(15).

Contudo, apesar do aparato tecnológico, são necessárias intervenções de enfermagem para a segurança do paciente, como o monitoramento dos sinais vitais e a observação de sinais e sintomas, ações que podem reduzir a ocorrência e intensidade da hipotensão durante a HD.

Dentre os incidentes ocorridos durante a prestação do cuidado, destacam-se os incidentes com cateter para HD, por incompatibilidade do acesso com o fluxo prescrito, 44 (86,28\%). Estudo realizado em UTI no Brasi|(5) identificou a predominância de uso do cateter de duplo lúmen (CDL) como via de acesso para a HD à beira leito, assim como o identificado neste estudo. Ainda, verificou-se $86,28 \%$ de incidentes relacionados à incompatibilidade do acesso CDL com o fluxo prescrito, o que poderia contribuir para a coagulação do $\mathrm{SE}^{(16)}$.

Os incidentes relacionados ao fluxo inadequado de sangue podem ser associados à condição clínica do paciente, habilidade técnica do profissional, tempo de permanência do cateter, posição inadequada da ponta do cateter ${ }^{(17)}$. A coagulação do SE, linhas e capilares pode ser devido à inadequação do funcionamento do cateter para $\mathrm{HD}^{(13)}$. A coagulação do SE representou $78(30,12 \%)$ dos incidentes classificados como inerentes ao tratamento identificados nesta pesquisa.

Pesquisa realizada com enfermeiros atuantes no cuidado a pacientes em HD contínua em UTI, identificou como principal incidente a perda de volume sanguíneo por não ser realizada a devolução do sangue ao paciente. Destaca-se, nesse estudo, a falta de habilidade do profissional de enfermagem em fazer a devolução do sangue quando a máquina alarmou indicando coagulação sanguínea ${ }^{(18)}$.

A falha em tecnologias disponibilizadas pela clínica foi o tipo de incidente envolvendo tecnologias de saúde com maior frequência, $105(66,46 \%)$, com destaque para vazamento da máquina de osmose reversa, 41 (39,05\%), e falta de água três (60,00\%). Foi identificado em estudo realizado em hospital sentinela do Brasil $2,2 \%$ EA relacionados à falha na distribuição de água ${ }^{(7)}$.

O tratamento e a qualidade da água são importantes, visto que se trata do maior insumo em HD. Garantir essa qualidade reduz a morbimortalidade e hospitalizações, necessitando, assim, de um sistema de vigilância ${ }^{(19)}$. Os gestores de serviço de diálise são responsáveis por esta atividade ${ }^{(19)}$.

Em 2010, o Ministério da Saúde lançou a Política Nacional de Gestão de Tecnologias em Saúde. Essa medida teve como objetivo ampliar a produção científica, de forma a subsidiar os gestores de serviços de saúde na aquisição e retirada de tecnologias em saúde ${ }^{(20)}$.

A dificuldade de apoio da equipe multiprofissional na ocorrência do incidente foi um achado importante, compreendendo 185 (58,73\%) notificações. Estudo realizado com equipe de enfermagem em um hospital sentinela de Goiânia afirmou que existem problemas de comunicação nos serviços de HD hospitalar e ambulatorial, elevando os riscos de $\mathrm{EA}^{(16)}$. 
ilegibilidade das prescrições de hemodiálise. Estudo de campo, aplicado e descritivo, realizado em 2017, mostrou que em 35\% das prescrições houve dificuldades de compreensão. A ilegibilidade pode comprometer o tratamento e a segurança do paciente, causando insuficiência do tratamento, complicações de saúde e óbito(21).

Assim como a falha na programação da máquina em relação à prescrição $(19,13 \%)$, estudo identificou $83,9 \%$ de falhas individuais de profissionais que geraram EA em pacientes que realizaram HD em um hospital sentinela de Goiânia ${ }^{(16)}$. A falta de atenção do profissional comprometeu a segurança do paciente, verificada em 5,9\% dos EA relatados.

Foi resultado dessa pesquisa o dimensionamento insuficiente da equipe de enfermagem e influência desse na ocorrência das circunstâncias notificáveis. Observa-se que ocorreram mais incidentes quando a equipe estava realizando mais de doze horas de atividades contínuas. Estudo realizado em cidades europeias verificou que longas jornadas de trabalho associadas à implementação de horas extras resultaram em assistência de baixa qualidade ao paciente ${ }^{(22)}$.

O dimensionamento da equipe deve levar em consideração a complexidade do cenário, o envolvimento de cuidados simultâneos, quantidade de pacientes, demanda do cuidado, etapa do ciclo de vida, contingências e a demanda de cuidados em enfermagem ${ }^{(23)}$. As instituições de saúde possuem como desafio a necessidade de adaptação à evolução das particularidades do ambiente, por meio de gestão de recursos humanos que sejam concomitantemente seguras, eficientes e centradas no paciente ${ }^{(23)}$.

Os EA inerentes ao tratamento apresentaram percentual de $39,61 \%$, enquanto que os EA evitáveis estiveram presentes em $15,81 \%$ das notificações. Pesquisa transversal realizada em HD identificou, em maior frequência, EA relacionados ao fluxo sanguíneo inadequado, enquanto que no presente estudo o EA mais identificado foi a hipotensão(7).

Programa inovador de capacitação envolvendo equipe de enfermagem e supervisores, nas áreas de desenvolvimento de segurança do paciente, resultou em melhora significativa nessa área, contudo, identificou como áreas frágeis da cultura de segurança a resposta não punitiva frente aos erros e subnotificações de incidentes ${ }^{(24)}$. É importante destacar que a OMS adota como premissa a não culpabilização do profissional que cometeu o erro( ${ }^{(25)}$.

No presente estudo, houve baixa frequência de notificação de alguns incidentes, o que pode ser justificado por serem relatadas em instrumento próprio para notificação de incidentes e a ausência de rotinas preestabelecidas para a notificação. São possíveis causas para a subnotificação: as dificuldades do profissional de cumprir a rotina de notificar, a sensação da não melhoria dos processos após notificar, a ausência do anonimato e confidencialidade, medo da resposta negativa da equipe de trabalho, medo da punição, dentre outras ${ }^{(26)}$.

O estudo apresentou limitações, como a dificuldade do profissional técnico em enfermagem da clínica em acessar os prontuários dos pacientes que receberam prescrição de HD. Devido à fonte de dados ser secundária e a análise retrospectiva, a ausência de informações dificultou ou inviabilizou a análise de alguns incidentes notificados. Contudo, espera-se que os achados discutidos neste estudo possam fomentar novas propostas de melhoria de gestão e consequente melhoria na qualidade do serviço prestado aos pacientes que realizam HD à beira leito.

\section{CONCLUSÃO}

Os incidentes atingiram em maior frequência homens, idosos, diabéticos. Foram frequentes os procedimentos de HD por meio de cateter para HD, na modalidade de terapia 
SLED, com tempo de sessão entre quatro e cinco horas, sem heparina e sessão subsidiada por convênios não-SUS. Os incidentes com maior frequência foram a hipotensão e falhas em tecnologias de saúde. Esses geraram atraso para o início do tratamento e requereram vigilância.

Os profissionais que identificaram os incidentes, na maioria, foram os técnicos em enfermagem da clínica que administra a HD à beira leito. Eles registraram dificuldades de apoio da equipe multiprofissional e as circunstâncias notificáveis ocorreram predominantemente quando o técnico estava exercendo mais de 12 horas de trabalho ininterruptas.

Foram identificados incidentes do tipo circunstância notificável, seguidos de EA inerentes ao tratamento, que podem ser minimizados por meio de melhorias nas ações de gerenciamento do cuidado durante sessões de HD à beira leito.

A análise dos tipos de incidentes permite que estudantes e expertises da área subsidiem estratégias para a melhoria do serviço. Para além do local estudado, o conhecimento da realidade e proposição de melhorias da segurança do paciente fomentam a formulação de estratégias com ações visionárias de prevenção desses incidentes na área de nefrologia. Desse modo, foram levantadas sugestões e propostas em conjunto com profissionais da empresa frente aos dados encontrados. A exemplo, ajustes importantes no dimensionamento dos técnicos de enfermagem e na manutenção preventiva dos equipamentos.

A HD à beira leito é uma terapia complexa, que pode gerar EA graves ao paciente. Por isso, é necessário rever a gestão da qualidade dos processos e riscos que envolvem essa terapia, formulando barreiras efetivas para os incidentes, revisando protocolos e rotinas constantemente.

\section{REFERÊNCIAS}

1. Organização Mundial da Saúde - OMS. Estrutura conceitual da classificação Internacional sobre segurança do paciente. [Internet] Lisboa: OMS; 2011. [acesso em 22 abr 2021]. Disponível em: https:// apps.who.int/iris/handle/10665/70882.

2. Couto RC, Pedrosa TMG, Roberto BAD, Daibert PB, Instituto de Estudos de Saúde Suplementar - IESS. Anuário da segurança assistencial hospitalar no Brasil. [Internet] IESS; 2017 [acesso em 06 fev 2018]. Disponível em: https://www.iess.org.br/cms/rep/anuario atualizado 0612.pdf.

3. Novaretti MCZ, Santos E de V, Quitério LM, Daud-Gallotti RM. Sobrecarga do trabalho da enfermagem e incidentes e eventos adversos em pacientes internados em UTI. Rev Bras Enferm [Internet]. 2014 [acesso em 06 fev 2018]; 67(5). Disponível em: http://www.redalyc.org/pdf/2670/267032830004.pdf.

4. Luft J, Boes AA, Lazzari DD, Nascimento ERP do, Busana J de A, Canever BP. Lesão renal aguda em unidade de tratamento intensivo: características clínicas e desfechos. Cogitare enferm [Internet]. 2016 [acesso em 20 out 2018]; 21(2). Disponível em: http://dx.doi.org/10.5380/ce.v21i2.43822.

5. Sesso RC, Lopes AA, Thomé FS, Lugon JR, Martins CT. Inquérito Brasileiro de Diálise Crônica. J Bras Nefrol [Internet]. 2016 [acesso em 21 fev 2018]; 39(3). Disponível em: http://doi.org/10.5935/01012800.20170049.

6. Bray BD, Boyd J, Daly C, Donaldson K, Fox JG, Innes A, et al. How safe is renal replacement therapy? A national study of mortality and adverse events contributing to the death of renal replacement therapy recipients. Nephrol. Dial. Transplant. [Internet]. 2014 [acesso em 14 fev 2018]; 26(3). Disponível em:

https://doi.org/10.1093/ndt/gft197. 
7. Sousa MRG de, Silva AEB de C, Bezerra ALQ. Prevalência de eventos adversos em uma unidade de hemodiálise. Rev Enferm UERJ [Internet]. 2016 [acesso em 19 nov 2018]; 24(6). Disponível em: https://doi. org/10.12957/reuerj.2016.18237.

8. World Health Organization (WHO). Minimal information model for patient safety incident reporting and learning systems. [Internet]. Geneva: WHO; 2016 [acesso em 16 out 2018]; Disponível em: https://apps. who.int/iris/handle/10665/255642.

9. Pérez MA, Iglesias MG, Sánchez IP, Ruiz GC, Solanas MC, López EA. Análisis del registro de un sistema de notificación de incidentes en una unidad de cuidados críticos. Enferm. Intensiva [Internet]. 2016 [acesso em 07 fev 2018]; 27(3). Disponível em: https://doi.org/10.1016/j.enfi.2015.12.003.

10. Siman AG, Brito MJM. Changes in nursing practice to improve patient safety. Rev Gaucha Enferm [Internet]. 2017 [acesso em 28 set 2018]; 37(spe). Disponível em: https://doi.org/10.1590/19831447.2016.esp.68271.

11. International Society of Nephrology (ISN). Acute Kidney Injury [Internet]. Bruxelas: ISN; 2018 [acesso em 05 dez 2018]. Disponível em: https://www.theisn.org/topics/acute-kidney-injury.

12. Borges F, Bohrer CD, Bugs TV, Nicola AL, Tonini NS, Oliveira JLC de. Dimensionamento de pessoal de enfermagem na UTI- adulto de hospital universitário público. Cogitare enferm [Internet]. 2017 [acesso em: 2018 dez 10]; 22(2). Disponível em: http://dx.doi.org/10.5380/ce.v22i2.50306.

13. Aguiar LL, Guedes MVC, Oliveira RM, Leitão IMT de A, Pennafort VP dos S, Barros AA. Enfermagem e metas internacionais de segurança: avaliação em hemodiálise. Cogitare enferm [Internet]. 2017 [acesso em 14 fev 2018]; 22(3). Disponível em: http://dx.doi.org/10.5380/ce.v22i3.45609.

14. Gomes ET, Nascimento MJS dos S. Assistência de enfermagem nas complicações durante as sessões de hemodiálise. Enfermagem Brasil. [Internet]. 2018 [acesso em 12 dez 2018]; 17(1). Disponível em: https://doi.org/10.33233/eb.v17i1.1127.

15. Morton PG, Fontaine DK. Cuidados críticos de enfermagem: uma abordagem holística. 9. ed. Rio de Janeiro: Guanabara Koogan; 2011.

16. Sousa MRG de, Silva AEB de C, Bezerra ALQ, Freitas JS de, Miasso Al. Adverse events in hemodialysis: reports of nursing professionals. Rev. esc. enferm. USP. [Internet] 2013 [acesso em 27 mai 2013];47(1). Disponível: http://dx.doi.org/10.1590/S0080-62342013000100010.

17. Ferreira V, Andrade D de. Catheters for hemodialysis: a reality report. Medicina (Ribeirão Preto). [Internet]. 2007 [acesso em 19 fev 2016]; 40(4). Disponível em: https://www.revistas.usp.br/rmrp/\%20 article/view/355/356.

18. Andrade BRP. O enfermeiro e o paciente em hemodiálise contínua na UTI: manejo da tecnologia na perspectiva da segurança [dissertação]. Rio de Janeiro (RJ): Escola de Enfermagem Ana Nery/UFRJ; 2016.

19. Jesus GP de, Almeida AA. Principais problemas gerados durante a terapia de hemodiálise associados à qualidade da água. Rev atual saúde. [Internet]. 2016 [acesso em 17 mar 2019]; 3(3). Disponível em: http://atualizarevista.com.br/article/principais-problemas-gerados-durante-a-terapia-de-hemodialiseassociados-a-qualidade-da-agua-v-3-n-3/.

20. Ministério da Saúde (BR). Política Nacional de Gestão de Tecnologias em Saúde. [Internet]. Brasília: Ministério da Saúde; 2010 [acesso em 14 dez 2018]; Disponível em: https://bvsms.saude.gov.br/bvs/ publicacoes/politica nacional gestao tecnologias saude.pdf.

21. Silva DX da, Cruz JM de L, Silva LM, Avelino LPB, Torres LA, Barbosa Neto PA. Legibilidade informacional: análise da clareza na apresentação das informações em receituários médicos. Revista Brasileira de Biblioteconomia e Documentação. [Internet]. 2017 [acesso em 18 mar 2018]; 13(esp.). Disponível em: https://febab.emnuvens.com.br/rbbd/article/view/992/1029.

22. Griffiths P, Dall'Ora C, Simon M, Ball J, Lindqvist R, Rafferty AM, et al. Nurses' shift length and overtime working in 12 European countries: the Association with perceived quality of care and patient 
safety. Medical Care [Internet]. 2014 [acesso em 10 dez 2018]; 52(11). Disponível em: http://dx.doi. org/10.1097/MLR.0000000000000233.

23. Carlesi KC, Padilha KG, Toffoletto MC, Henriquez- Roldán C, Juan MAC. Ocorrência de incidentes de segurança do paciente e carga de trabalho de enfermagem. Rev Latino-Am Enfermagem [Internet]. 2017 [acesso em 10 dez 2018]; 25:e2841. Disponível em: http://doi.org/10.1590/1518-8345.1280.2841.

24. Amiri M, Khademian Z, Nikandish R. The effect of nurse empowerment educational program on patient safety culture: a randomized controlled trial. BMC Medical Education [Internet]. 2018 [acesso em 10 dez 2018]; 18(158). Disponível em: https://doi.org/10.1186/s12909-018-1255-6.

25. Alves EAV, Sousa P, Faria PL. Desafios jurídicos do reporte de eventos adversos: proposta de agenda para melhoria da segurança do paciente. In: Santos AO, Lopes LT, editors. Coletânea direito à saúde: institucionalização. Brasília: Conselho Nacional de Secretários de Saúde - CONASS; 2018. p. 295-306.

26. Faria PL, Moreira PS, Pinto LS. Direito e segurança do paciente. In: Sousa P, Mendes W, editors. Segurança do paciente: conhecendo os riscos nas organizações de saúde. Rio de Janeiro: Editora Fiocruz; 2014. p. 115-34. 


\title{
INCIDENTES EM SESSÕES DE HEMODIÁLISE À BEIRA LEITO EM UNIDADES DE TERAPIA INTENSIVA
}

\section{RESUMO:}

Objetivo: analisar incidentes durante sessões de hemodiálise à beira leito, realizadas em Unidades de Terapia Intensiva. Método: estudo transversal, retrospectivo, desenvolvido em Unidades de Terapia Intensiva de Goiânia, Brasil. Foi realizada análise de incidentes identificados em sessões de hemodiálise à beira leito, entre março e abril de 2018. Foi realizada análise descritiva para os dados categóricos e para os contínuos, média e desvio-padrão da média. Resultados: foram analisadas 873 sessões e registrados 563 incidentes. Dentre os notificados, 259 (46\%) foram inerentes ao tratamento. O near miss foi o tipo de incidente de maior frequência, gerando atraso no início da sessão, e não obtiveram apoio da equipe multiprofissional $(p<0,05)$. Em $132(56,12 \%)$ das circunstâncias notificáveis, o profissional trabalhava por mais de 12 horas ininterruptas $(p<0,05)$. Conclusão: a análise dos resultados permite que estudantes e expertises da área subsidiem estratégias de melhoria para o serviço.

DESCRITORES: Qualidade da Assistência à Saúde; Vigilância de Evento Sentinela; Segurança do Paciente; Dano ao Paciente; Enfermagem em Nefrologia.

\section{INCIDENCIAS EN LAS SESIONES DE HEMODIÁLISIS A PIE DE CAMA EN LAS UNIDADES DE CUIDADOS INTENSIVOS}

\section{RESUMEN:}

Objetivo: analizar las incidencias durante las sesiones de hemodiálisis a pie de cama, realizadas en las Unidades de Cuidados Intensivos. Método: estudio transversal, retrospectivo, desarrollado en Unidades de Cuidados Intensivos de Goiânia, Brasil. Entre marzo y abril de 2018 se realizó un análisis de las incidencias identificadas en las sesiones de hemodiálisis a pie de cama. Se realizó un análisis descriptivo para los datos categóricos y para los continuos, la media y la desviación estándar de la media. Resultados: Se analizaron 873 sesiones y se registraron 563 incidentes. Entre los notificados, 259 (46\%) eran inherentes al tratamiento. El cuasi accidente fue el tipo de incidente más frecuente, generando retraso en el inicio de la sesión, y no obtuvo apoyo del equipo multiprofesional ( $p<0,05)$. En $132(56,12 \%)$ de las circunstancias notificables, el profesional trabajó más de 12 horas ininterrumpidas $(p<0,05)$. Conclusión: el análisis de los resultados permite que los estudiantes y los expertos del área establezcan estrategias de mejora para el servicio.

DESCRIPTORES: Calidad de la Atención de Salud; Vigilancia de Guardia; Seguridad del Paciente; Daño del Paciente; Enfermería en Nefrología.

*Artigo extraído da dissertação de mestrado "Incidentes em Sessões de Hemodiálise à Beira Leito em Unidades de Terapia Intensiva". Universidade Federal de Goiás, 2019.

Recebido em: 09/09/2020

Aprovado em: 26/02/2021

Editora associada: Juliana Balbinot Reis Girondi

\author{
Autor Correspondente: \\ Natália Nunes Costa \\ Universidade Federal de Goiás - Goiânia, GO, Brazil \\ E-mail: natynatyufg@gmail.com
}

Contribuição dos autores:

Contribuições substanciais para a concepção ou desenho do estudo; ou a aquisição, análise ou interpretação de dados do estudo - Costa MM da, Schincaglia RM, Freitas NR de, Costa CL, Suzuki K; Elaboração e revisão crítica do conteúdo intelectual do estudo - Barreto R dos SS; Responsável por todos os aspectos do estudo, assegurando as questões de precisão ou integridade de qualquer parte do estudo - Costa NN. Todos os autores aprovaram a versão final do texto.

Copyright @ 2021 Este é um artigo em acesso aberto distribuído nos termos da Licença Creative Commons Atribuição, que permite o uso irrestrito, a distribuição e reprodução em qualquer meio desde que o artigo original seja devidamente citado. 